\title{
Diseño e integración del recurso multimedia «mine-ducation» en Educación Primaria: Valoraciones aportadas por expertos
}

\author{
María Celia Etchegaray Centeno $\left({ }^{*}\right)$, Ana María Duarte Hueros $\left({ }^{* *}\right)$ y María Dolores Guzmán Franco $\left({ }^{* *}\right)$ \\ (*) Universidad Caece - Argentina ${ }^{(*}$ ) Universidad de Huelva - España
}

\section{RESUMEN}

El estudio describe y analiza el proceso seguido para la evaluación y validación a través de la técnica del juicio de expertos de un novedoso recurso educativo implementado en Educación Primaria: «mine-ducation». Se trata de un sistema multimedia en línea que tiene en cuenta las inteligencias múltiples y los estilos de aprendizaje del alumnado para brindar la posibilidad de una enseñanza individualizada. Se emplea un método no experimental, utilizando la técnica de juicio de expertos y las entrevistas individuales semiestructuradas para la recogida de datos. El análisis se aborda siguiendo de forma sistemática, secuencial y ordenada, los procesos recomendados en el método comparativo constante para la codificación abierta, axial y selectiva. Los resultados obtenidos permiten identificar que los expertos entrevistados perciben la herramienta como una aliada en la docencia, entre otros motivos porque facilita el proceso de enseñanza-aprendizaje acorde con las necesidades del alumnado y las nuevas tendencias pedagógicas en relación con las TIC. Además, es valorado por proveer información sobre el perfil cognitivo de cada estudiante, facilitando una educación más individualizada. Las dificultades que los docentes encuentran para implementar este recurso nos hacen pensar que los centros escolares se encuentran en proceso de adaptación metodológica y tecnológica.

Palabras Clave: Tecnologías de la Información y Comunicación (TIC), educación, multimedia, juicio de expertos, inteligencias múltiples, educación personalizada.

\section{«Mine-ducation» design and integration in primary education: experts assessment}

\section{ABSTRACT}

This study describes and analyses the process of expert assessment and validation of «mine-ducation», a new educational resource for primary education. This online multimedia system is designed to taken into account the multiple levels of intelligence and learning styles of students, and provides the opportunity for individualized teaching. A non-experimental method consisting of individual semi-structured interviews of experts was applied to gather data for a systematic, sequential and ordered analysis, as the process recommended in the constant comparative method for open, axial and selective coding. The results show that the experts see the tool as a useful aid to teaching, as it empowers the teaching-learning process when providing for individual student needs, and it fits in with new pedagogical trends in ICT. The experts also praised the system for supplying information on each student's cognitive profile, which enables a more individualized form of education. However, difficulties encountered in implementing this resource reveal that education centres are still in a process of methodological and technological adaptation.

Keywords: ICT, education, multimedia, expert judgment, multiple levels, personal education.

\section{Introducción}

Son numerosos los cambios que se vienen reclamando al mundo educativo para adaptarse a las nuevas demandas y desafíos de la sociedad actual y futura. Las competencias y habilidades que necesitan ser desarrolladas en la sociedad del conocimiento han cambiado, demandando nuevas y diferentes formas de enseñar. Como señalaban Ananiadou y Claro (2009):
La evolución de la sociedad y la economía exige que los sistemas educativos doten a los jóvenes de nuevas habilidades y competencias que les permitan beneficiarse de las nuevas formas de socialización y contribuir activamente al desarrollo económico en un sistema donde el principal activo es el conocimiento. (p. 5)

Esta idea, ampliamente compartida por la comunidad educativa (Bates, 2015; Camps, 2009; Dede, 2010; García-Ruíz, Ramírez y Rodríguez, 2014; Hargreaves y Fullan, 2014; Scott, 2015), y tam- 
bién la base de múltiples estudios e investigaciones, tanto a nivel nacional como internacional, tales como DeSeCo (Definición y Selección de las Competencias) de la OCDE realizado a finales de los años 90, de acuerdo con el cual, las competencias clave pueden aglutinarse en tres grandes categorías, la primera de ellas, relacionada con el uso de las tecnologías de la información y la comunicación (TIC); junto con la interacción entre grupos heterogéneos; y saber actuar de forma autónoma (Rychen, 2003).

$Y$ es que estamos siendo testigos de una revolución en el aprendizaje, de una necesidad de innovación y de renovación de la educación, donde el apoyo tecnológico sea la herramienta para brindar una educación que permita descubrir y desarrollar el talento de cada persona.

En la revolución del aprendizaje que deviene de incorporar las TIC en el aula, una de las oportunidades que estas ofrecen es la de crear una nueva experiencia de enseñanza y de aprendizaje personalizada, participativa y colaborativa (Coll, 2008; Salinas, 2004).

Cuando se habla de enseñanza personalizada, se hace referencia a respetar los ritmos y las diferencias individuales, adaptando la enseñanza a cada persona, de la manera que necesite, motive y prefiera, siendo requisito previo necesario, el reconocimiento de las diferentes capacidades individuales al momento de aprender, así como sus diferentes estilos de aprendizaje.

Capacidades individuales que pueden ser desarrolladas, como es el caso de las inteligencias múltiples propuestas originariamente por Howard Gardner. De acuerdo con el planteamiento del autor, la inteligencia es «un potencial biopsicológico para procesar información que puede ser activado en un entorno cultural para resolver problemas o crear productos que son de valor en una cultura» (Gardner y Moran, 2006, p. 227). De forma que la inteligencia, como potencial, no sería innata y fija, sino que puede ser desarrollada y moldeada. Además, al igual que hay muchos tipos de problemas a resolver, hay muchos tipos de inteligencia, identificando, al menos, ocho grandes tipos de inteligencias: lógica-matemática, lingüística, espacial, musical, corporal-kinestésica, naturalista, intrapersonal, e interpersonal (Gardner y Hatch, 1989; Gardner, 1995; 2006).

A pesar de que esta teoría ha tenido muchas críticas desde un sector de la comunidad científica (controversia en la que no se va a entrar en estos momentos por falta de espacio si bien, remitimos al lector interesado a la revisión crítica realizada por Cuevas, 2015 y Navarrete y Queutre, 2015). Sin embargo, debemos reconocer que se trata de un planteamiento que pone de relieve la existencia de diferentes y diversas capacidades o habilidades y estrategias de aprendizaje.

Diferentes estudios y revisiones de experiencias empíricas han puesto en evidencia la valía de estos planteamientos (Armstrong, 2000; Gouws, 2007; Pérez y Beltrán, 2006) al informarnos que ante la diversidad de formas de aprender y enseñar se incrementa la posibilidad de mejorar el rendimiento académico. Corroborando la necesidad de valorarlas de forma previa a la puesta en marcha de cualquier programa educativo, constituyendo un interesante pilar para la toma de decisiones en relación con la diversificación de contenidos y estrategias didácticas a movilizar (Díaz, Varela y Rodríguez, 2017; Emst, 2001).

En lugar de ignorar o negar estas diferencias bajo la creencia de que todos los estudiantes tienen o deben tener, las mismas dotes y habilidades mentales, el sistema educativo deberá proveer a cada estudiante con oportunidades para el aprendizaje que maximicen sus potencialidades intelectuales (Emst, 2001, p.329-330).

Muy relacionado con esas diferentes capacidades o inteligencias, se encuentran los estilos o formas de aprendizaje. Estilos que han sido estudiados desde muy variados enfoques y perspectivas proponiendo diversos instrumentos y métodos de evaluación diferentes, como ponen de relieve los autores consultados (Alonso, Gallego y Honey, 1994; García, Antonio y Gutié-

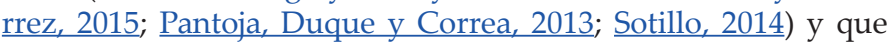
reafirman que no existe una sola y única manera de aprender, sino que depende de una multiplicidad de factores de diversa índole tanto biológica, como personal, social y contextual.

Siguiendo a Kolb (1971) se identifican cuatro estilos de aprendizaje: estilo activo (predominante en las personas que aprenden mejor, haciendo); estilo reflexivo (aprenden a través de la observación y el análisis); estilo teórico (predominante en personas que aprenden mejor a través de la lógica, análisis y síntesis de información); y estilo pragmático (personas que aprenden a través de la prueba y puesta en práctica constante).

Los estilos de aprendizaje tienen implicaciones educativas por cuanto, al presentar información mediante diferentes estrategias pedagógicas, teniendo en cuenta los diferentes estilos de aprendizaje se favorece el éxito académico (Gallego, 2013; Sarasin, 2006). En esta línea, se sitúan investigaciones realizadas en los últimos años en relación con el diseño, producción y evaluación de materiales y recursos digitales adaptados a los diferentes estilos de aprendizaje (Cabero, 2003; Dunn, Griggs, Gorman, Olson y Beasley: 1995; Tocci: 2013). Como afirma Pinto (2011):

La calidad aplicada a la información digital es una meta que implica un proceso continuo de planificación, análisis, diseño, implementación, promoción e innovación, para asegurar que la información cubra las necesidades de formación y aprendizaje de los usuarios en cuanto a contenido, presentación y usabilidad. (p. 209)

\subsection{Planteamiento del problema}

Tomando en consideración el marco teórico que lo sustenta, se desarrolla el software «mine-ducation». Se trata de un sistema multimedia online mediante el cual se pueden tomar cursos de diferentes temáticas, con la particularidad que el contenido del curso se personaliza según las preferencias de aprendizaje de cada estudiante (inteligencias múltiples y estilos de aprendizaje) al momento de recibir la teoría, realizar las prácticas y las evaluaciones. También el sistema permite registrar las acciones realizadas por el alumnado a medida que avanza en el curso, con el objetivo de brindar al docente información acerca de sus preferencias de navegación y poder realizar un análisis de su posible incidencia en la motivación y el rendimiento académico. La temática seleccionada del curso para probar el funcionamiento del recurso educativo es «uso responsable de Internet y redes sociales», tema que las autoras consideran de gran importancia y relevancia actual, orientado a niños/as entre 9 y 11 años.

La necesidad de realizar una evaluación formal del sistema multimedia desarrollado y de las nuevas estrategias educativas que ofrece, nos llevó a desarrollar la investigación evaluativa con los expertos que describimos a continuación, como fase previa a la versión final del multimedia para su utilización con la población destinataria.

\section{Objetivos del estudio}

El objetivo general del estudio ha sido evaluar el recurso «mine-ducation» desde una perspectiva técnica, didáctica y comunicativa, de acuerdo con la opinión de los expertos.

Centrándose esta evaluación, en cuatro grandes dimensiones:

- Aspectos técnicos del software en términos de calidad de los componentes multimedia, velocidad de procesamiento y presencia/ausencia de errores técnicos. 
- Características pedagógicas, en términos de adecuación del contenido a los objetivos didácticos, respeto a las diferencias individuales, motivación que genera y métodos de ejercitación y evaluación que facilita.

- Aspectos de diseño y estética en términos de simplicidad y claridad de su interfaz gráfica y multimedia, navegabilidad y diversidad de su diseño comunicativo.

- Aspectos funcionales del sistema en términos de usabilidad.

\section{Metodología}

Para desarrollar la investigación, se opta por una metodología de corte cualitativo, utilizando la técnica de valoración de expertos a través de entrevista individual semiestructurada. Decidimos realizar el estudio desde una perspectiva cualitativa por cuanto nuestra intención era recabar información en profundidad sobre posibles soluciones y modificaciones del sistema producido. Desde esta perspectiva, se selecciona la técnica de juicio de expertos por ser una estrategia bastante usual para la evaluación de materiales de enseñanza en el terreno de la investigación educativa (Area y Guarro, 2012; Cabero y Barroso, 2013), y porque consideramos que los expertos podían proporcionarnos valoraciones fiables del sistema, al tiempo que ofrecer «recomendaciones en función de un máximo de competencia» (Mengual, 2011, p. 158).

Por ello, nos decantamos por la realización de entrevistas individuales semiestructuradas, con objeto de indagar amplia y profundamente en la valoración del recurso por parte de profesionales de los campos de la educación, seguridad informática y psicopedagogía, en representación de los diferentes sectores implicados en el diseño, producción y uso de recursos multimedia educativos como «mine-ducation» y a partir de su interacción con el sistema multimedia.

\subsection{Participantes}

Con el fin de obtener información suficiente desde diferentes perspectivas técnicas, psicopedagógicas y comunicativas, y proporcionar mayor riqueza al análisis del discurso, se tuvieron en cuenta los siguientes indicadores para la selección de los expertos participantes:

- Nivel educativo: maestros/as y psicopedagogos/as en activo, así como profesores/as universitarios/as expertos en tecnología educativa.

- Perfil académico: expertos en educación, psicopedagogía, tecnología y comunicación con orientación a la seguridad informática.

- Prestigio profesional: Se trata de profesionales con un profundo conocimiento del ámbito docente, académico o comunicativo en el que se desenvuelven, con una dilatada experiencia y un alto, o al menos considerable reconocimiento profesional en sus ámbitos de trabajo.

El panel de expertos quedó conformado por 8 profesionales, de los cuales, 1 estaba especializado en comunicación y seguridad informática; 2 en educación primaria; 3 en el ámbito de la psicopedagogía y 2 ejercían como profesores en contexto universitario.

\subsection{Fases de la investigación}

El desarrollo del estudio se organizó en tres fases consecutivas: 1) Planificación y diseño del juicio de expertos; 2) Experimentación de la evaluación; 3) Análisis de los datos y conclusiones.

\subsubsection{Planificación y diseño de la evaluación a expertos}

En esta fase se planificó la experimentación y definió la metodología de la investigación. Se elaboró y validó el protocolo guía de la entrevista; se prepararon las condiciones para llevar adelante la evaluación: selección y contacto a expertos/as y creación de un ambiente de prueba, denominado ambiente de testeo, donde los/as expertos/as podían ingresar y navegar en un curso que contiene formato, contenido y condiciones idénticas al que utilizarían posteriormente los alumnos/as.

\subsubsection{Elaboración del protocolo de entrevista}

El instrumento seleccionado para llevar a cabo la recogida de información fue la entrevista semiestructurada. Se diseñó un protocolo de entrevista conformado por una serie de temas eje y preguntas sugeridas, que sirvieron como guía para expertos/ as e investigadores/as al momento de desarrollar la entrevista, así como facilitar las tareas de análisis de los datos. Para elaborar este protocolo nos basamos en experiencias e investigaciones previas y revisamos modelos de evaluación de software educativo realizada por Cova, Arrieta y Aular (2008), y el instrumento de evaluación online realizado por Miller y Bach (2001).

\subsubsection{Experimentación de la evaluación}

Una vez que los expertos habían realizado un proceso de inmersión y familiarización con el recurso «Mine-education», obteniendo la misma experiencia a la que accederán alumnos/as y docentes, procedimos a la realización de las entrevistas personales grabadas, previo consentimiento de los/as entrevistados/as, para el posterior análisis de los datos.

\section{Resultados}

Para realizar el análisis de los datos, tomamos como referencia la metodología de la teoría fundamentada (Grounded Theory) desarrollada por Glaser y Strauss (Strauss y Corbin, 2002) muy utilizada en múltiples investigaciones (Álvarez, 2003; Andreu, García y Pérez, 2007; Dick, 2005; Meza, Torres y Lara, 2016; San Martín, 2014).

Se aborda el análisis realizando de forma sistemática, secuencial y ordenada los procesos recomendados por Strauss y Corbin (2002) utilizando el método comparativo constante. El procesamiento de los datos se ha realizado con asistencia del programa ATLAS.ti, (versión 7.5.7). A continuación, se exponen y analizan algunos de los resultados más sobresalientes para nuestro estudio.

Hemos de indicar que las categorías y códigos obtenidos, se sometieron a control a través de la doble codificación de las grabaciones por parte de dos investigadoras diferentes, teniendo un alto índice de fiabilidad al observarse una alta concordancia entre los analistas.

Una vez transcritas las grabaciones, se realizó una lectura minuciosa y reiterada de la totalidad de la información, codificando los textos de acuerdo con los tópicos centrales del estudio, resaltando los elementos relevantes del discurso de los entrevistados, e identificando posibles categorías y códigos emergentes del mismo.

De forma paralela se iba construyendo una matriz de análisis desarrollada en un libro o sistema de códigos para el análisis de los datos conforme iban surgiendo en las entrevistas.

En dicho libro de códigos se incluía una descripción detallada de cada código, criterios de inclusión y exclusión, y ejemplos de texto real para cada tema. 
El resultado de este proceso fue un conjunto de códigos iniciales que se organizaron por dimensiones, dando lugar a cate- gorías iniciales y subcategorías. En la siguiente tabla 1, se recoge la matriz de análisis con las categorías y códigos resultantes.

Tabla 1. Matriz de análisis resultante de la codificación abierta

\begin{tabular}{|c|c|}
\hline Dimensión & Códigos y Familias de códigos \\
\hline \multirow[t]{2}{*}{ Técnica } & Funcionamiento técnico (Tiempo de Respuesta, Errores técnicos...) \\
\hline & Calidad multimedia (calidad de imágenes, audios, videos...) \\
\hline \multirow[t]{2}{*}{ Diseño y estética } & Navegabilidad (simplicidad, coherencia, claridad, adaptabilidad...) \\
\hline & $\begin{array}{l}\text { Gráfica y multimedia (colores, tipografía, animaciones, imágenes estáticas, herramientas de } \\
\text { diseño multimedia, vídeo, música, audio...) }\end{array}$ \\
\hline \multirow[t]{9}{*}{ Contenidos } & Pertinencia (nivel de adecuación a la edad y preferencias tecnológicas) \\
\hline & Extensión (adecuación) \\
\hline & Información (nivel de actualización, precisión y exhaustividad...) \\
\hline & Lenguaje (estilo, forma, tonalidad y grado de pertinencia al público objetivo) \\
\hline & Léxico (comprensibilidad) \\
\hline & Ortografía (errores orto gramaticales) \\
\hline & $\begin{array}{l}\text { Contenidos inclusivos (diversidad de género, étnico/racial, contravalores, adecuación a la } \\
\text { cultura argentina) }\end{array}$ \\
\hline & Ejemplos (adecuación a la edad, nivel de realismo, nivel de actualización) \\
\hline & Aplicabilidad (obstaculizada, facilitada...) \\
\hline \multirow[t]{11}{*}{ Pedagógica } & Motivación (facilitada o no facilitada...) \\
\hline & Nivel comunicativo (claridad en los objetivos, instrucciones y consignas de ejercicios...) \\
\hline & Evaluación (nivel de dificultad, valoración general de la actividad) \\
\hline & Ejercitación (respeto a las diferencias individuales, edad, actitudes a fomentar) \\
\hline & Repetición (nivel de repetición) \\
\hline & Estructuración curso (organización y orden) \\
\hline & Participación (nivel de actividad que promueve...) \\
\hline & Requisitos alumnado (nivel de atención y concentración...) \\
\hline & Refuerzo (grado de comunicación sobre logros de aprendizaje) \\
\hline & Personajes (nivel lúdico, adecuación, diversidad, emociones que fomentan) \\
\hline & Interacción (nivel de interactividad, causas que la facilitan) \\
\hline \multirow[t]{7}{*}{ Funcional } & Usabilidad (formas de uso, modalidades de enseñanza, accesibilidad) \\
\hline & Restricciones de uso (causas, dificultades) \\
\hline & Rol familia (necesidades de participación y supervisión...) \\
\hline & Autonomía percibida (obstáculos y facilidades) \\
\hline & Rol docente (formas de participación) \\
\hline & Funcionalidades propuestas (aspectos de mejora) \\
\hline & Valoración general (cualidades del sistema) \\
\hline
\end{tabular}

Una vez realizada esta primera parte del análisis (codificación abierta), se da comienzo al proceso de búsqueda de relaciones entre las diferentes categorías y subcategorías a través de diagramas en los que se enlazan las categorías de acuerdo con sus propiedades y dimensiones. En el caso que aquí se trata, se diferencia en la codificación entre categorías positivas frente a aquellas que habían obtenido valoraciones negativas.

Del análisis global se observa que la categoría más referenciada es la de «ejercitación» lo que nos remite al respeto de las preferencias del alumnado al darles la posibilidad de elegir el formato y el estilo preferente para la ejercitación. Esta posibili- dad que brinda la práctica hizo que el sistema fuera valorado por su carácter innovador y útil en términos de un alto potencial de usabilidad, al poder usar la herramienta para conocer las preferencias del alumno. Otra de las categorías con alta frecuencia es la gráfica y multimedia lo que ha ocasionado que el sistema sea considerado dinámico en términos de interactividad y riqueza multimedia.

En el siguiente diagrama conceptual (figura 1), se expresan estas relaciones entre categorías y códigos con mayor frecuencia y en la tabla 2, se puede ver la lista completa de las mismas. 
Figura 1. Mapa conceptual del análisis global

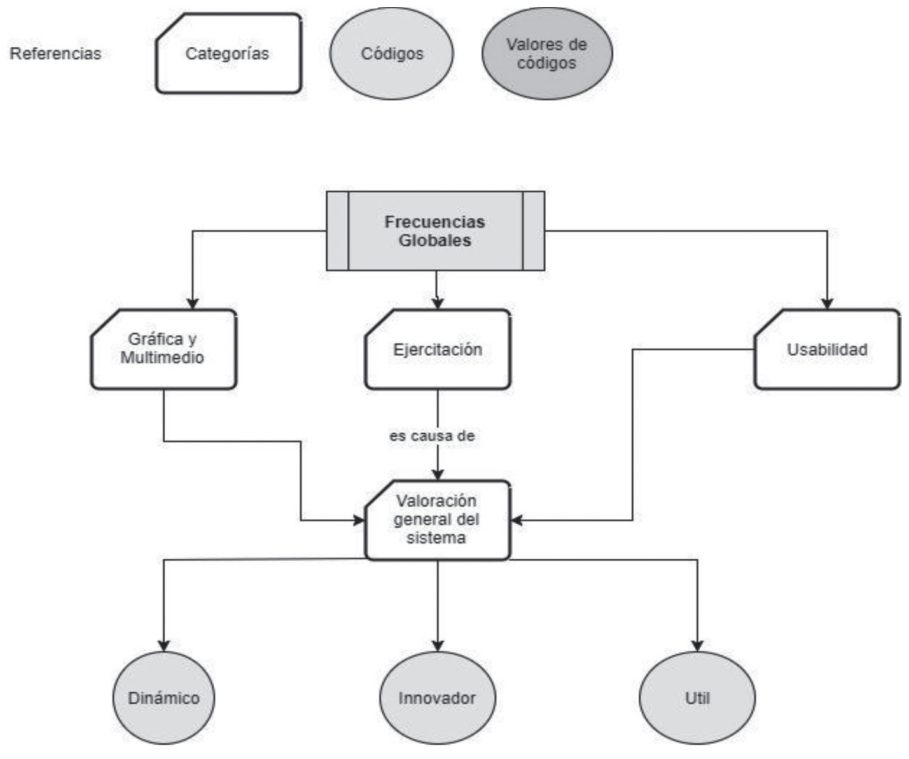

Tabla 2. Frecuencias y porcentajes de las categorías sobre el total

\begin{tabular}{|c|c|c|}
\hline Categorías & $F$ & $\%$ \\
\hline Ejercitación & 37 & 9,59 \\
\hline Valoración general & 33 & 8,55 \\
\hline Usabilidad & 31 & 8,03 \\
\hline Gráfica y multimedia & 24 & 6,22 \\
\hline Contenido: información & 22 & 5,70 \\
\hline Rol del Profesor & 21 & 5,44 \\
\hline Contenido: pertinencia de la edad & 20 & 5,18 \\
\hline Interactividad & 19 & 4,92 \\
\hline Navegabilidad & 19 & 4,92 \\
\hline Propuestas para futuras versiones & 17 & 4,40 \\
\hline Personajes & 17 & 4,40 \\
\hline Contenido: lenguaje & 15 & 3,89 \\
\hline Funcionamiento técnico & 13 & 3,37 \\
\hline Restricciones de Usos & 12 & 3,11 \\
\hline Contenido: extensión & 12 & 3,11 \\
\hline Nivel comunicativo & 11 & 2,85 \\
\hline Contenido: aplicabilidad & 10 & 2,59 \\
\hline Participación & 9 & 2,33 \\
\hline Contenido: ejemplos & 8 & 2,07 \\
\hline Contenido: inclusivo & 8 & 2,07 \\
\hline Evaluación & 8 & 2,07 \\
\hline Rol de los Padres & 5 & 1,30 \\
\hline Autonomía percibida & 5 & 1,30 \\
\hline Aspectos que necesita desarrollar el alumnado & 4 & 1,04 \\
\hline Motivación & 3 & 0,78 \\
\hline Calidad multimedia & 1 & 0,26 \\
\hline Estructura del curso & 1 & 0,26 \\
\hline Repetición & 1 & 0,26 \\
\hline Totales & 386 & 100,00 \\
\hline
\end{tabular}

Aula Abierta, volumen 47, nº 3, julio-septiembre, 2018, págs. 307-318 


\subsection{Aspectos positivos del recurso multimedia educativo}

En una primera aproximación, y haciendo un cómputo general de las unidades textuales que se refieren a los aspectos positivos y negativos del recurso educativo, el profesorado entrevistado identifica más aspectos positivos que negativos, en concreto 190 referencias textuales se refieren a los aspectos positivos, frente a 70 que aluden aspectos negativos, lo que representa el $73 \%$ frente al $27 \%$ de las intervenciones en este sentido.

De forma resumida, podemos indicar que los principales aspectos positivos que los expertos atribuyen al recurso educativo se relacionan con la «Ejercitación: positiva» (posibilidad de elegir el tipo de ejercicio a realizar relacionándolo con sus preferencias y estilos de aprendizaje; así como el canal por el que recibir el mensaje (voz, texto, imagen); y con la familia de códigos relacionadas con la «Gráfica y multimedia: positiva».

De hecho, los expertos valoran en primer lugar la adecuación a las preferencias multicanales de los/as alumnos/as de esa edad y la música utilizada; así como todo el material es valorado por su calidad y dinamismo. Por ejemplo, el entrevistado no 5 aseveraba:
Magnífica presentación, musicalización a lo largo del proyecto. La animación es acorde a la edad de los chicos (Entrevista $\mathrm{n}^{\mathrm{O}} 5$ ).

También la actualidad e interés de los temas que se trabajan, así como la importancia de que los niños y niñas de primaria sepan hacer un uso responsable de Internet y las redes sociales son cuestiones más valoradas. Siendo destacables también las referencias positivas al nivel de interactividad y a su nivel técnico.

Considero que el contenido es actual, son cosas que les suceden a los chicos cuando empiezan a navegar en Internet. Las recomendaciones son muy atinadas. En sí está muy bien construido, con cosas interesantes e importantes para los niños, que orientan al niño sobre qué hacer y qué no en Internet, qué está bien y qué no está bien, las normas y buenas prácticas de seguridad (Entrevista $\mathrm{n}^{\mathrm{o}} 2$ ).

En la siguiente tabla $n^{\circ} 3$, se recogen las frecuencias, promedios ponderados y porcentajes totales, obtenidos en las diferentes categorías relacionadas con los aspectos positivos del recurso.

Tabla 3. Aspectos positivos de recurso educativo multimedia

\begin{tabular}{|c|c|c|c|}
\hline Categorías codificadas & $\mathrm{F}$ & $\% \mathrm{P}$ & $\%$ \\
\hline Ejercitación: positiva & 29 & 15,03 & 11,03 \\
\hline Gráfica y multimedia: positiva & 19 & 9,84 & 7,22 \\
\hline Contenido: información: positivo & 17 & 8,81 & 6,46 \\
\hline Contenido: pertinencia de la edad: positivo & 16 & 8,29 & 6,08 \\
\hline Contenido: lenguaje: positivo & 14 & 7,25 & 5,32 \\
\hline Interactividad: positiva & 13 & 6,74 & 4,94 \\
\hline Personajes: positivo & 13 & 6,74 & 4,94 \\
\hline Funcionamiento técnico: positivo & 10 & 5,18 & 3,80 \\
\hline Navegabilidad: positiva & 10 & 5,18 & 3,80 \\
\hline Participación: positiva & 9 & 4,66 & 3,42 \\
\hline Contenido: inclusivo: positivo & 8 & 4,15 & 3,04 \\
\hline Contenido: aplicabilidad: positivo & 7 & 3,63 & 2,66 \\
\hline Contenido: ejemplos: positivo & 7 & 3,63 & 2,66 \\
\hline Evaluación: positivo & 7 & 3,63 & 2,66 \\
\hline Nivel comunicativo: positivo & 5 & 2,59 & 1,90 \\
\hline Motivación: positiva & 3 & 1,55 & 1,14 \\
\hline Autonomía percibida: positiva & 2 & 1,04 & 0,76 \\
\hline Contenido: extensión: positivo & 1 & 0,52 & 0,38 \\
\hline Calidad multimedia: positiva & 1 & 0,52 & 0,38 \\
\hline Estructura del curso: positiva & 1 & 0,52 & 0,38 \\
\hline Repetición: positiva & 1 & 0,52 & 0,38 \\
\hline TOTAL & 193 & 100,00 & 73,38 \\
\hline
\end{tabular}

La figura 2 es una representación gráfica de las categorías y subcategorías relacionadas de acuerdo con los aspectos positivos destacados del análisis 
Figura 2. Diagrama categorías y códigos - valoraciones positivas

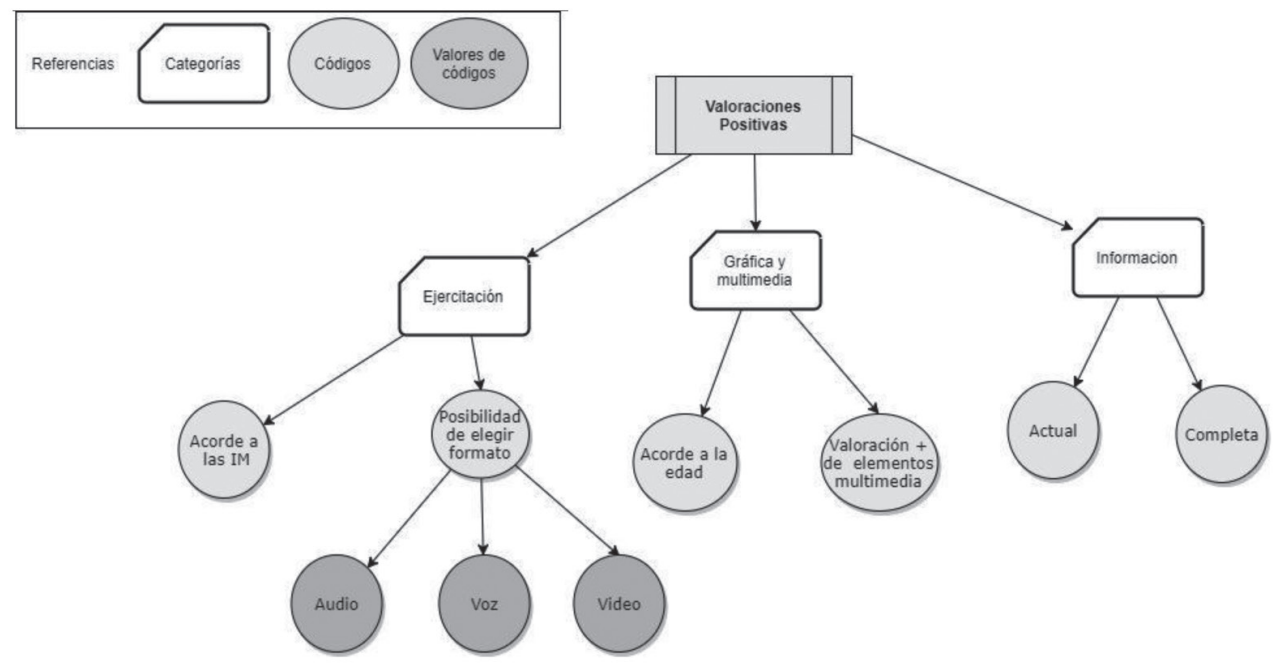

\subsection{Aspectos negativos y propuestas de mejora del recurso multimedia educativo}

Si bien los/as expertos/as detallan mayoritariamente aspectos positivos del sistema, se describen también algunos aspectos negativos. De forma resumida, podemos indicar que los principales aspectos negativos que los expertos atribuyen al recurso educativo se relacionan con «Contenido: extensión» (duración total de cada unidad con respecto al tiempo escolar); con la familia de códigos relacionadas con la «Navegabilidad»y, con la «Ejercitación».

Los aspectos negativos de la extensión se asocian con una de las restricciones de uso del sistema que los expertos han encontrado en términos de limitaciones en la duración del tiempo de clase y en los periodos atencionales de la edad.

Partiendo de la realidad que tenemos día a día, con respecto a dar las clases el tiempo es un gran limitante, porque muchas veces no podemos trabajar ciertos temas o no podemos llevar a cabo ciertos proyectos justamente por la falta de tiempo (Entrevista 5).

Utilice la modalidad auditiva, y me costó sostenerlo tanto tiempo. Teniendo en cuenta que los periodos atencionales en esta nueva generación se caracterizan por ser cortos, considero que es un punto a analizar (Entrevista 6).

La navegabilidad se asocia a las restricciones de uso por cuestiones madurativas del alumnado al momento de acceder a enlaces externos al sistema:

Lo que requiere el sistema, de anotar el score, cuesta un poco para el niño. Se podría perder el hilo (Entrevista 1).

En cuanto a la ejercitación, solicitan en algunos casos puntuales aumentar la dificultad y agregar validaciones para evitar la posibilidad de cometer fraudes por parte de los alumnos.

A lo mejor si despliegas primero un renglón después otro y otro, te obligaría a escribir tres consejos porque ahora con escribir una letra ya te libera el nivel y te permite pasar a la siguiente etapa (Entrevista 8).

Las cuestiones madurativas del niño hacen necesario incluir en los ejercicios del sistema a los padres con el fin de integrar lo virtual y lo real y de hacerlos partícipes de la educación de sus hijos.

Que el alumnado pueda aplicar todo esto que se dicta en el curso, hay que sacarlo del sistema, tratar de que más allá de las actividades del sistema, que el niño pueda hablar con el padre para traspasar el plano de lo teórico. Integrar lo virtual con lo real... (Entrevista 1).

Otra de las restricciones de uso mencionadas es la escasa infraestructura tecnológica de algunas escuelas, sobre todas las de gestión pública, como se puede apreciar en la siguiente referencia:

Me parece que podría ser usado en escuelas privadas, que tienen acceso a computadoras e Internet, o en escuelas secundarias del estado que son las que tienen PC. Esta escuela primaria donde yo trabajo todavía no tiene las PC del estado nacional (Entrevista 4).

También se destaca la importancia de que el profesorado tenga un rol como persona responsable de desarrollar el aprendizaje del alumnado y de guía en el uso de la herramienta.

Un coaching inicial se necesitaría para animarlos a vivenciar un proceso que va a ser individual pero también está compartido con el grupo. Me parece que está bueno. Donde se plantea inicialmente los objetivos del curso y dónde tuviera un rol el profe o sino lo único que hace es evaluar (Entrevista 8).

En la Tabla 4, se recogen las frecuencias, promedios ponderados con relación a las valoraciones negativas, y porcentajes sobre el total, obtenidos en las diferentes categorías relacionadas con los aspectos negativos del recurso. En la figura 3 se pueden ver a modo resumen los aspectos negativos destacados.

Tabla 4. Aspectos negativos de recurso educativo multimedia

Categorías codificadas
Contenido: extensión: negativo
Navegabilidad: negativa
Ejercitación: negativa
Interactividad: negativa

$\begin{array}{ccc}\text { F } & \% \text { P } & \% \\ 11 & 15,71 & 4,18 \\ 9 & 12,86 & 3,42 \\ 8 & 11,43 & 3,04 \\ 6 & 8,57 & 2,28\end{array}$


Categorías codificadas

\begin{tabular}{ccc} 
F & $\%$ P & $\%$ \\
6 & 8,57 & 2,28 \\
5 & 7,14 & 1,90 \\
5 & 7,14 & 1,90 \\
4 & 5,71 & 1,52 \\
4 & 5,71 & 1,52 \\
3 & 4,29 & 1,14 \\
3 & 4,29 & 1,14 \\
3 & 4,29 & 1,14 \\
1 & 1,43 & 0,38 \\
1 & 1,43 & 0,38 \\
1 & 1,43 & 0,38 \\
0 & 0,00 & 0,00 \\
0 & 0,00 & 0,00 \\
0 & 0,00 & 0,00 \\
0 & 0,00 & 0,00 \\
0 & 0,00 & 0,00 \\
0 & 0,00 & 0,00 \\
70 & 100,00 & 26,62 \\
\hline & &
\end{tabular}

TOTAL

100,00

26,62

Figura 3. Diagrama categorías y códigos - valoraciones negativas

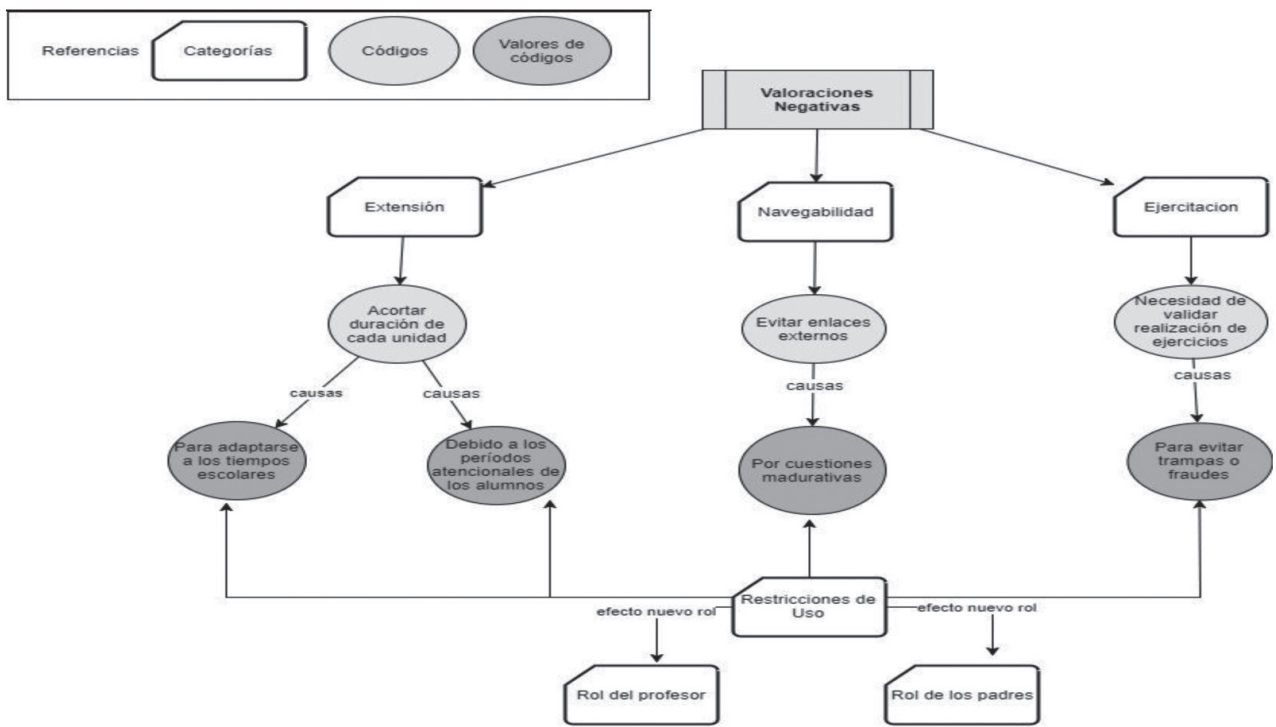

\subsection{Análisis relacional entre las diferentes categorías analizadas}

Como último paso en el análisis realizado, se considera importante indagar sobre las relaciones que se producen entre las diferentes categorías y subcategorías identificadas. Para ello se halló la adición del total de frecuencias de cada categoría (tanto aspectos positivos como negativos), y se calculó el porcentaje de incidencia de los aspectos positivos y negativos sobre la frecuencia total de cada categoría. Finalmente se comparan los porcentajes obtenidos para conocer qué aspecto predomina en cada categoría. Toda esta información se detalla en la siguiente tabla.

Tabla 5. Comparativa de categorías indicadoras

\section{Categoría}

Autonomía percibida

Calidad multimedia

Aplicabilidad
$\%$ Positivo \% Negativo

(F/Total categoría)

40

100

70
(F/Total categoría)

0

30
\%Positivo - \%Negativo

$-20$

100

40
Comparativa

Negativa

Positiva

Positiva 


$\begin{array}{ccccc}\text { Categoría } & \begin{array}{c}\text { \% Positivo } \\ \text { (F/Total categoría) }\end{array} & \begin{array}{c}\text { \% Negativo } \\ \text { (F/Total categoría) }\end{array} & \text { \%Positivo - \%Negativo } & \text { Comparativa } \\ \text { Ejemplos } & 88 & 13 & 75 & \text { Positiva } \\ \text { Extensión } & 8 & 92 & -84 & \text { Negativa } \\ \text { Contenidos inclusivos } & 100 & 0 & 100 & \text { Positiva } \\ \text { Información } & 77 & 23 & 54 & \text { Positiva } \\ \text { Lenguaje } & 93 & 7 & 86 & \text { Positiva } \\ \text { Pertinencia } & 80 & 20 & 60 & \text { Positiva } \\ \text { Ejercitación } & 78 & 22 & 56 & \text { Positiva } \\ \text { Estructuración del curso } & 100 & 0 & 100 & \text { Positiva } \\ \text { Evaluación } & 88 & 13 & 75 & \text { Positiva } \\ \text { Funcionamiento técnico } & 77 & 23 & 54 & \text { Positiva } \\ \text { Gráfica y multimedia } & 79 & 21 & 58 & \text { Positiva } \\ \text { Interacción } & 68 & 32 & 36 & \text { Positiva } \\ \text { Motivación } & 100 & 0 & 100 & \text { Positiva } \\ \text { Navegabilidad } & 53 & 47 & 6 & \text { Positiva } \\ \text { Nivel comunicativo } & 45 & 55 & -10 & \text { Negativa } \\ \text { Participación } & 100 & 0 & 100 & \text { Positiva } \\ \text { Personajes } & 76 & 24 & 52 & \text { Positiva } \\ \text { Repetición } & 100 & 0 & 100 & \text { Positiva } \\ \text { Promedio } & 77 & 23 & 54 & \end{array}$

Al analizar los datos obtenidos, se observa que solamente 3 indicadores tienen un porcentaje negativo mayor al positivo. Éstos son los indicadores de "autonomía percibida», «contenido: extensión» $y$ «nivel comunicativo», mientras que todos los demás indicadores tienen un porcentaje positivo mayor al $40 \%$ como se observa en la tabla anterior.

En términos generales, las valoraciones positivas superan ampliamente a las negativas en todos los indicadores, siendo la valoración positiva total promedio de $77 \%$ y la negativa de $23 \%$, lo que nos permite considerar que el recurso cuenta con calidad técnica,

de diseño y estética, así como en aspectos pedagógicos y funcionales, de acuerdo con la opinión de los expertos consultados.

En la siguiente figura 4, se ha recogido un diagrama para representar estos resultados. En el mismo hemos marcado con color gris claro los indicadores con valoraciones positivas superior al $40 \%$ y en gris oscuro los tres indicadores con valoraciones negativas. Se aprecia en el mismo cómo la calidad del sistema predomina en los aspectos técnicos y de diseño y estética, teniendo cuestiones de calidad a revisar en los aspectos pedagógicos $\mathrm{y}$ funcionales.

Figura 4. Diagrama general de los aspectos de calidad analizados

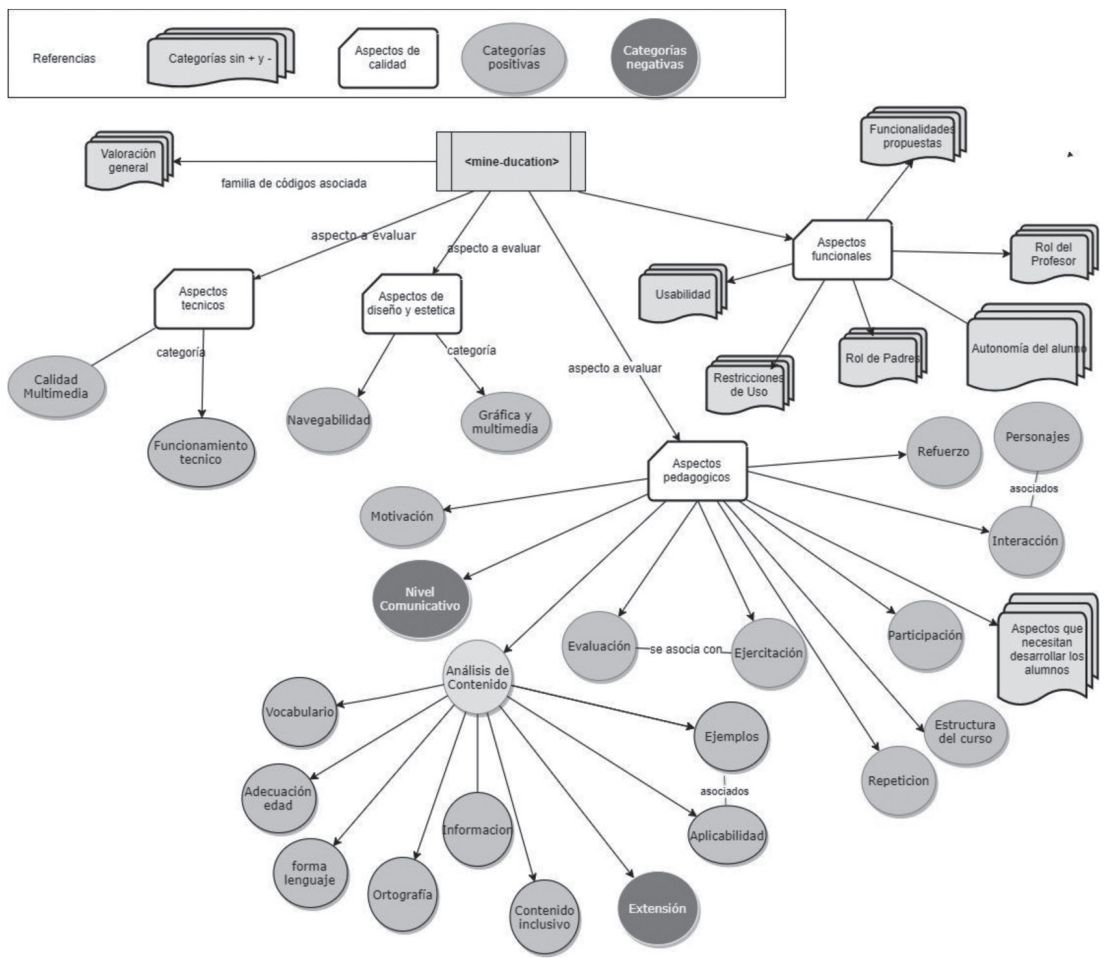




\section{Conclusiones}

De acuerdo con el análisis realizado, podemos afirmar que los/as expertos/as encuentran al sistema «mine-ducation», dinámico, multimedia e interactivo, innovador, facilitador de una educación personalizada, y útil al permitir al estudiante adecuarse a sus preferencias y estilos de aprendizaje, siendo un complemento muy interesante a la clase presencial. Si se contrastan las cuatro categorías que influyen en la eficacia del e-learning, según Kozlowski y Bell (2007): a) el nivel de riqueza del contenido, b) el nivel de realismo para favorecer la inmersión, c) el nivel de interactividad, y d) el nivel de comunicación que se posibilita; concluimos que las valoraciones hechas por los expertos sobre el recurso evaluado abarcan las categorías mencionadas que influyen en la eficacia del mismo.

Además de la evaluación positiva del software en sus diferentes aspectos según los términos de calidad considerados en el apartado correspondiente, queremos destacar que no ha recibido ninguna valoración negativa el contenido del curso en términos de discriminación o contravalores, en la reiteración a los/as alumnos/ as de los conceptos, en la calidad de sus elementos multimedia, en la estructura y organización del curso ni tampoco en su capacidad de motivar y de hacer participar activamente al alumnado.

Sin embargo, el uso del sistema puede verse restringido por cuestiones de los tiempos escolares, por la posibilidad de fraudes de los alumnos y por la falta de recursos económicos y tecnológicos de algunos colegios de primaria.

Vemos importante a partir del análisis de los resultados obtenidos, la necesidad de incorporar en futuras versiones del recurso educativo, nuevas funcionalidades que promuevan la integración de las tareas de planificación y también de ejecución en el docente. Siendo el encargado de generar su propio contenido, las actividades, las evaluaciones y también planificar las estrategias de aprendizaje de los estudiantes según su conocimiento del alumnado. Pudiendo además valorar el propio docente, las bondades de las pruebas previas sobre estilos y preferencias de aprendizaje, conformándose el sistema como herramienta de apoyo a su didáctica y a sus mejores prácticas.

Apoyos: Este trabajo está avalado por el Proyecto I+D+I, titulado "Competencias mediáticas de la ciudadanía en medios digitales emergentes (smartphones y tablets): prácticas innovadoras y estrategias educomunicativas en contextos múltiples" con clave EDU2015-64015-C3-1-R (MINECO/FEDER), financiado por el Fondo Europeo de Desarrollo Regional (FEDER) y Ministerio de Economía y Competitividad de España.

\section{Referencias}

Alonso, C. M., Gallego, D. y Honey, P. (1994). Los Estilos de Aprendizaje: procedimientos de diagnóstico y mejora. Bilbao: Ediciones Mensajero. Universidad Deusto.

Álvarez, J. L. (2003). Cómo hacer investigación cualitativa. Fundamentos y metodología. México: Paidós.

Ananiadou, K. y Claro, M. (2009). 21st Century Skills and Competences for New Millennium Learners in OECD Countries. OECD Education Working Papers, 41, OECD Publishing. doi: $10.1787 / 218525261154$

Andreu, J., García, A. y Pérez, A. (2007). Evolución de la teoría fundamentada como técnica de análisis cualitativo. Madrid: CIS.

Area, M. y Guarro, A. (2012). La alfabetización informacional y digital: fundamentos pedagógicos para la enseñanza y el aprendizaje competente. Revista Española de Documentación Científica, monográfico, 46-74. doi: 10.3989/redc.2012.mono.97
Armstrong, T. (2000). Las Inteligencias Múltiples en el aula: guía práctica para educadores. Buenos Aires: Editorial Manantial.

Bates, A.W. (2015). Teaching in a Digital Age: Guidelines for Designing Teaching and Learning. Vancouver BC: Tony Bates Associates Ltd.

Cabero, J. (2003). Replanteando la tecnología educativa. Comunicar, 21, 23-30. Recuperado de: https://bit.ly/2m76L4r

Cabero, J. y Barroso, J. (2013). La utilización del juicio de experto para la evaluación de TIC: el coeficiente de competencia experta. Bordón, 65(2), 25-38. doi: 10.13042/brp.2013.65202

Camps, V. (2009). La educación en medios, más allá de la escuela. Comunicar, 32, 139-145. doi: 10.3916/c32-2009-02-012

Coll, C. (2008). Aprender y enseñar con las TIC. Expectativas, realidad y potencialidades. Boletín de la Institución Libre de Enseñanza, 72, 17-40.

Cova, Á.; Arrieta, X. y Aular, J. (2008). Revisión de modelos para evaluación de software educativos. Télématique, 7(1), 94-116. Recuperado de: https://bit.ly/2zqsVbl

Cuevas, J. (2015). Is learning styles-based instruction effective? A comprehensive analysis of recent research on learning styles. Theory and Research in Education, 13(3), 308-333. Doi: $0.1177 / 1477878515606621$

Dede, C. (2010). Comparing frameworks for 21st century skills. In J. Bellanca \& R. Brandt. (Eds.) 21st century skills: Rethinking how students learn, 20, 51-76. Bloomington, IN: Solution Tree Press

Díaz, L.E; Varela, S.P. y Rodríguez, L.P. (2017). Multiple intelligences and curriculum implementation: Progress, trends and opportunities, Revista Psicodidáctica, 22(1), 69-83. doi: 10.1016/S1136-1034(17)30046-1

Dick, B. (2005). Grounded theory: a thumbnail sketch. Recuperado de: https://bit.ly/2L2bhzp

Dunn, R., Griggs, S. A., Gorman, B. S., Olson, J. y Beasley, M. (1995). A Meta-Analytic Validation of the Dunn and Dunn Model of Learning-Style Preferences. The Journal of Educational Research, 88(6), 353-362. doi: 10.1080/00220671.1995.9941181

Emst, G. (2001). Educación para todos: La Teoría de las Inteligencias Múltiples de Gardner. Revista de Psicología de la PUCP, 19(2), 320-322. Recuperado de: https://bit.ly/2JauGJi

Gallego, D.J. (2013). Ya he diagnosticado el estilo de aprendizaje de mis alumnos y ahora ¿Qué hago? Revista de Estilos de Aprendizaje, 6(12), 1-15. Recuperado de: https://bit.ly/2KYpeyu

García, J. L., Antonio, P. y Gutiérrez, M. (2015). (2015). Estilos de Aprendizaje y su relación con el instrumento Egel-Ceneval. Journal of Learning Styles, 8(16), 212-250. Recuperado de: https://bit.ly/2L5IO8L

García, R., Ramírez, A. \& Rodríguez, M.M. (2014). Media Literacy Education for a New Prosumer Citizenship. [Educación en alfabetización mediática para una nueva ciudadanía prosumidora]. Comunicar, 43, 15-23. doi: 10.3916/C43-2014-01

Gardner, H. y Moran, S. (2006). The Science of Multiple Intelligences Theory: a response to Lynn Waterhouse. Educational Psychologist, 41 (4), 227-232. doi: 10.1207/s15326985ep4104_2

Gardner, H. (1995). Are There Additional Intelligences? The Case for the Naturalist Intelligence. Harvard Project Zero. Cambridge, MA: President and Fellows of Harvard College.

Gardner, H. (2006). Multiple Intelligences: New Horizons. New York: BasicBooks.

Gardner, H. y Hatch, T. (1989). Multiple Intelligences go to school. Educational Researcher, 18(8), 4-1.

Gouws, F.E. (2007). Teaching and learning through multiple intelligences in the outcomes-based education classroom, Africa Education Review, 4:2, 60-74. doi: 10.1080/18146620701652705

Hargreaves, A. y Fullan, M. (2014). Capital profesional. Transformar la enseñanza en cada escuela. Madrid: Ediciones Morata. 
Kolb, D. A. (1971). The Learning Style Inventory: Technical Manual. Boston MA: McBer \& Co.

Kozlowski, S. W. J. y Bell, B. S. (2007). A theory-based approach for designing distributed learning systems. In S. M. Fiore \& E. Salas (Eds.). Toward a science of distributed learning, 15-39. Washington, DC: APA.

Mengual, S. (2011). La importancia percibida por el profesorado y el alumnado sobre la inclusión de la competencia digital en educación Superior. Alicante: Departamento de Didáctica General y Didácticas específicas de la Facultad de Alicante.

Meza, L. D.; Torres, S. A. y Lara, J. J. (2016). Estrategias de aprendizaje emergentes en la modalidad e-learning. Revista de Educación a Distancia, 48(5). Recuperado de: https://bit.ly/2zqbeJ7

Miller, K. y Bach, J. (2001). SAS in School. Recuperado de: https:// bit.ly/2JbiSXD

Navarrete, P. y Queutre, J.L. (2015). Teoría de las Inteligencias Múltiples en Educación: una revisión crítica. doi: 10.13140/ RG.2.1.5182.0245

Pantoja, M. A., Duque, L. I. y Correa, J. S. (2013). Modelos de estilos de aprendizaje: una actualización para su revisión y análisis. Revista Colombiana de Educación, 64, 79-105. Recuperado de: https://bit.ly/2uneeA8

Pérez, L. y Beltrán, J. (2006). Dos décadas de «inteligencias múltiples»: implicaciones para la Psicología de la Educación. Papeles del Psicólogo, 27(3), 147-164.

Pinto, M. (2010). Evaluación y mejora de la calidad de los recursos educativos electrónicos en el ámbito universitario español desde un enfoque documental. Ibersid, № 3, 105-116. Recuperado de: https://bit.ly/2u9kSux
Rychen, S. (2003). A Frame of Reference for Defining and Selecting Key Competencies in an International Context. In S. Rychen; L. H. Salganik \& McLaughlin, M.E. (Eds.). Definition and Selection of Key Competencies. Contributions to the Second DeSeCo Symposium, 109-116. Recuperado de: https://bit. ly/2NH8eLt

Salinas, J. (2004). Cambios metodológicos con las TIC. Estrategias didácticas y entornos virtuales de enseñanza-aprendizaje. Bordón, 56(3-4), 469-481.

San Martín, D. (2014). Teoría fundamentada y Atlas.ti: recursos metodológicos para la investigación educativa. Revista Electrónica de Investigación Educativa, 16(1), 103-122. Recuperado de: https://bit.ly/2NHPzz0

Sarasin, L.C. (2006). Learning Styles Perspectives. Impact in the classroom. Madison, WI: Atwood Publishing.

Scott, C. L. (2015). The futures of learning-3: What kind of pedagogies for the 21st century? UNESCO Education Research and Foresight, Paris. [ERF Working Papers Series, 15]. Recuperado de: https://bit.ly/2pQuFke

Sotillo, J. F. (2014) El Cuestionario CHAEA-Junior o cómo diagnosticar el estilo de aprendizaje en alumnos de Primaria y Secundaria. Journal of Learning Styles, 7(13), 182-201. Recuperado de: https://bit.ly/2u80Uk1

Strauss, A. y Corbin, J. (2002). Bases de la investigación cualitativa. Técnicas y procedimientos para desarrollar la Teoría Fundamentada. Medellín: Universidad de Antioquia.

Tocci, A.M. (2013) Estilos de aprendizaje de los alumnos de ingeniería según la programación neuro-lingüística. Revista Estilos de Aprendizaje, 11(12), 1-20. Recuperado de: https://bit. ly/2JcHrDh 
